\title{
Erratum to: Same-day discharge after craniotomy for supratentorial tumour surgery: a retrospective observational single-centre study
}

Lashmi Venkatraghavan, MD • Suparna Bharadwaj, MD • Karolyn Au, MD •

Mark Bernstein, MD • Pirjo Manninen, MD

Published online: 4 January 2017

(C) Canadian Anesthesiologists' Society 2017

\section{Erratum to: Can J Anesth/J Can Anesth (2016) \\ 63:1245-1257 \\ DOI 10.1007/s12630-016-0717-8}

In the article entitled: "Same-day discharge after craniotomy for supratentorial tumour surgery: a retrospective observational single-centre study" published in the November 2016 issue of the Journal, Can J Anesth 2016; 63: 1245-1257, in Table 3, next to Osmotic therapy, the values between Inpatient and Same-day discharge have been corrected and are highlighted in bold. The publisher apologizes most sincerely for this error.

The online version of the original article can be found under doi:10.1007/s12630-016-0717-8.

L. Venkatraghavan, MD · S. Bharadwaj, MD .

P. Manninen, MD

Department of Anesthesia and Pain Management, Toronto

Western Hospital, University of Toronto, Toronto, ON, Canada

L. Venkatraghavan, MD ( $\square)$

Department of Anesthesia, Toronto Western HospitalUniversity

Health Network, University of Toronto, 399 Bathurst St,

Toronto, ON M5T 2S8, Canada

e-mail: lashmi.venkatraghavan@uhn.on.ca

K. Au, MD - M. Bernstein, MD

Division of Neurosurgery, Toronto Western Hospital, University

of Toronto, Toronto, ON, Canada 
Table 3 Perioperative management

\begin{tabular}{|c|c|c|c|}
\hline Perioperative management & $\begin{array}{l}\text { Inpatient } \\
(n=154)\end{array}$ & $\begin{array}{l}\text { Same-day discharge } \\
(n=175)\end{array}$ & $P$ value \\
\hline Monitoring & $103(67 \%)$ & $23(13 \%)$ & $<0.001$ \\
\hline Invasive arterial blood pressure & $6(3 \%)$ & $0(0 \%)$ & 0.01 \\
\hline Central venous pressure & $89(58 \%)$ & $11(6 \%)$ & $<0.001$ \\
\hline \multicolumn{4}{|l|}{ Urinary catheter } \\
\hline Intraoperative fentanyl use $(\mu \mathrm{g})$ & 177(89) & $81(56)$ & $<0.001$ \\
\hline Osmotic therapy & $15(10 \%)$ & $1(1 \%)$ & $<0.001$ \\
\hline Total fluid administered $(\mathrm{mL})$ & $2,370(430)$ & $1,345(665)$ & $<0.001$ \\
\hline Hypotension ( $>20 \%$ from baseline) & $106(69 \%)$ & $114(65 \%)$ & 0.48 \\
\hline Use of vasopressors & $85(55 \%)$ & $39(22 \%)$ & $<0.001$ \\
\hline Use of anti-hypertensive medication & $84(55 \%)$ & $8(5 \%)$ & $<0.001$ \\
\hline Estimated blood loss $>300 \mathrm{~mL}$ & $54(35 \%)$ & $6(3 \%)$ & $<0.001$ \\
\hline Use of colloid & $16(10 \%)$ & $1(1 \%)$ & $<0.001$ \\
\hline Blood transfusion & $7(4 \%)$ & $0(0 \%)$ & 0.004 \\
\hline Postoperative opioid consumption (morphine equivalent) (mg) & $23(16)$ & $16(17)$ & $<0.001$ \\
\hline Antiemetic use in PACU & $84(55 \%)$ & $39(22 \%)$ & $<0.001$ \\
\hline
\end{tabular}

All values are $n(\%)$ or mean (SD), where indicated; $n=$ number; PACU $=$ postanesthesia care unit 